\title{
Residential Area Surveillance and Parking System
}

\author{
Mathivanan. P, Amarnath. G, Anantharaman. R, Bharath Kumar. M, Joseph Charles. A
}

\begin{abstract}
The vehicles playing the vital role in our day to day life for transport, and some of the vehicles violates the traffic rules are also increasing, vehicle theft, unnecessary entering into highly restricted areas, increased number of accidents lead to increase in the rate of crime slowly. The vehicle had its own identity it should be recognized which plays the major role in the world. For recognition of the vehicles which are used commonly in the field of safety and security system, LPDR plays a major role and the vehicle registration number is recognized at some certain distance accurately. License Plate recognition is the most efficient and cost effective technique used for detection and recognition purposes. Automatic license plate recognition (ALPR) is used for finding the location of the license plate in the vehicle. These methods and techniques vary based on the conditions like, quality of the image, vehicle on a fine-tuned position, effects of lighting, type of image, etc. The objective is to design an efficient automatic conveyance identification system of sanctioned or unauthorized in the residential societies by utilizing the conveyance number plate. By getting the car image from the surveillance camera in the entrance, we recognizing the number plate and the characters are extracted using OCR (optical character recognition). It converts the character in the image to plain text. Then the plain text of the license plate is cross-verified with the database to check whether the vehicle belongs to residents or visitor. It sends the alert message to the security official when a new visitor request method in a residential area. The log details are stored separately for the resident and visitor in the database. It also provides the details about the parking area availability in the residential area. By calculating the number of vehicles in and out of the area, the detail or availability parking slot is displayed and it sis robust to the size, lighting effects with high rate of detection.
\end{abstract}

Keywords: Automatic number plate recognition, License plate detection recognition, Optical character recognition.

\section{INTRODUCTION}

Automatic Number Plate Recognition (ANPR) is a one of the image processing technology, where we can able to identify the vehicles by using the number (license) plate. After getting the image of the vehicle from the surveillance camera mounted in the entrance area the number plate is detected and it is recognized by the extracting the character from the detected number plate by OCR technique. It converts the optical character to plain text.

\section{Revised Manuscript Received on June 10, 2020.}

* Correspondence Author

P. Mathivanan*, Assistant Professor, Department of Information Technology, Manakula Vinayagar Institute of Technology, Puducherry, India. E-mail: mathi64it@gmail.com

G. Amarnath, Final Year, Department of Information Technology, Manakula Vinayagar Institute of Technology, Puducherry, India. E-mail: throneamar@gmail.com

R. Anantharaman, Final Year, Department of Information Technology, Manakula Vinayagar Institute of Technology, Puducherry, India. E-mail: anantharaman2207@gmail.com

Bharath Kumar. M, Final Year, Department of Information Technology, Manakula Vinayagar Institute of Technology, Puducherry, India.

Joseph Charles. A, Final Year, Department of Information Technology, Manakula Vinayagar Institute of Technology, Puducherry, India.

(c) The Authors. Published by Blue Eyes Intelligence Engineering and Sciences Publication (BEIESP). This is an open access article under the CC BY-NC-ND license (http://creativecommons.org/licenses/by-nc-nd/4.0/)
Then the plain text of the license plate is cross-verified with the database to check whether the vehicle belongs to residents or visitor. It sends the alert message to the security official when new visitor enters into a residential area. The log details are stored separately for the resident and visitor in the database. It also provides the details about the parking area availability inside the residential area. By calculating the number of vehicles in and out of the area, the detail or availability parking slot is displayed.

\section{LITERATURE REVIEW}

The Residential Area Surveillance system has been developed for different way of techniques to perform different technologies. Many references papers are taken from several case studies and it develop the different ways of technologies which are used for parking vehicles in automation. Most of the vehicle number plate detection process describe the license plate detection, Automatic license plate recognition, number plate detection and recognition of object, Optical character recognition are used for detecting the number plate as automatically.

This paper uses Yolo algorithm for object detection and object recognition. Yolo performs several innovations which increase accuracy of detecting and testing speed of a object. The primary purpose of this paper investigates some of another author and gives a disfigurement of the system proposed by them, provide a best solution for it.

\section{A. Efficient Scale-Adaptive License Plate Detection System}

This method proposes a scale-aware model which is coupled with a effective boosting algorithm, detect the number plate of various scale during the training period by learning the more important features of the various scales and reduce the detection time of the model searching in the unwanted areas. This proposed model is robust in terms of the scale perspective and quite efficient in various types of scenarios.

\section{B. New process of vehicle number plate location based} on YOLO-L model and plate pre-defined

This process is to detect the conveyance location of the license plate predicated on the new process YOLO model. This new process uses various mechanisms to detect and identify the vehicle and number plate area. It involves famous clustering method k-denotes++ to detect the effective number and recognize the best scale of license plate. And then, it verifies the structure and depth of process. It uses the YOLOv2 version. Number plate prior detection algorithm are acclimated to detect efficaciously distinguish number plates from single objects.

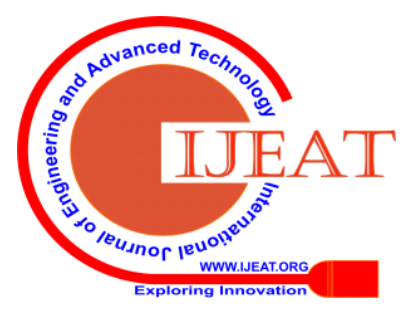




\section{An efficient way of Faster R-CNN for micro Object Detection}

The proposed method involves the fusion of the multi-scale convolution features from the inputs develop the feature map holds more details about the process, and utilize the suppression algorithm (Non maximum suppression) to reduce the loss due to the overlapping of the image or the object. The experimental has done on the algorithm in traffic sign detection. It shows that the algorithm has the good efficiency and performance. It has the high accuracy of the detection when compared to the other proposed methods. However it has the high performance it takes the more time on the experimental section.

\section{Faster R-CNN: Towards Real-Time Object Detection with Region Proposal Networks}

In this proposed method, a Region Proposal Network (RPN) which shares consummate object convolution features with the detection of object network, thus enabling approximately more effective region proposals. An Region proposal network is a planarity convolution network that detects bounds of the object and scores the unwanted area at each location of the object. The Region proposal network trained in the way to engender improved quality region proposals of a object, which are utilized by the Recursive Convolutional Neural Network for detection of object. We used to coalesce Expeditious R-CNN and RPN into a single network by utilizing their features-sharing.

\section{E. Object Detection Networks on Convolution Feature Maps}

It describes that, from a deep feature maps, a deep and convolution per-region classifier is of particular paramount for detection of a object, whereas latest superior image relegated into different models (such as GoogLeNets and ResNets) will not lead directly to good detection precision of object without utilizing such a per-region classifier.

\section{EXISTING SYSTEM}

\section{A. Scale-Aware License Plate Detection System}

The license plate detection is a effective approach in surveillance and vehicle identification technique. However there are several methods have been proposed in many ways, their idea and effectiveness is reduced to the categorical scenario and their performance dropping in more and different injuctively authorizing scenarios. The main problem to be addressed in this type of systems is the changing size of the license plates. This method proposes a scale-aware model which is coupled with a effective boosting algorithm, detect the number plate of various scale during the training period by learning the more important features of the various scales and reduce the detection time of the model searching in the unwanted areas. This proposed model is robust in terms of the scale perspective and quite efficient in various types of scenarios. However the method aware of scaling it has the less detection rate and accuracy is low when the object features are low.

\section{ARCHITECTURE DIAGRAM}

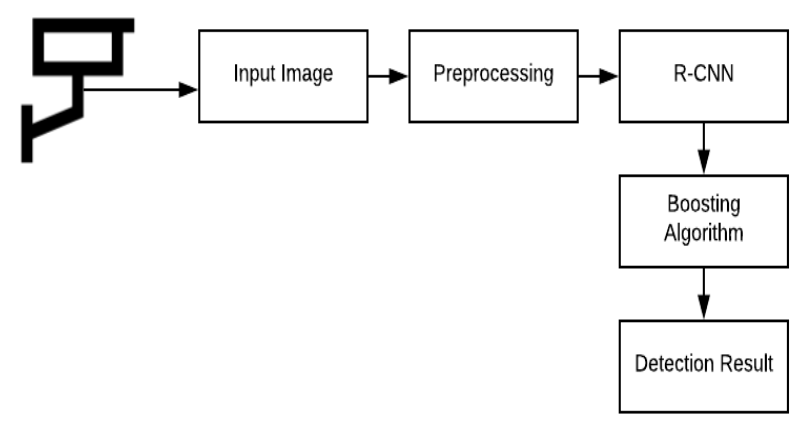

\section{DIS ADVANTAGES \\ Fig.1. Architecture of Existing System}

In the testing section, the existing object detector is robust to scaling, does not on the scales and performs well in few scenarios

$>$ It is proved that it has less performance when it comes to gradients.

$>$ It is not works well in many practical scenarios because of

$\circ$ Presence of noise

oBlurring.

\section{PROPOSED SYSTEM}

The recognition of the identity plays the major role in the authorization and surveillance areas. The identity of the automobiles can be recognized by number plates. They are the unique identity of the vehicle because each vehicle has its own registration number. It consists of the numbers and alphabets. The manual authorization of the vehicle identity by the human is quite intensive but the improved computational power of the systems can did the process easily for $24 * 7$.

Many of the methods in Automatic License plate recognition (ALPR) systems have constraints in their working scenarios, such as lighting, background conditions, skew angles of the detected number plate for recognition and distance between the subject and the camera. Hence the existing system used predefined methods for the recognition while setting up the environment.

In proposed work the objective of our work is to remove the limiting the environment with the predefined conditions, improving the rate of the detection to provide the faster detection result and recognition, robust to the jittering and blurring effects. Here we are using the YOLOv3 which has more potential to detect the object at the faster rate and perform the detection of the multiple instance of the same object.

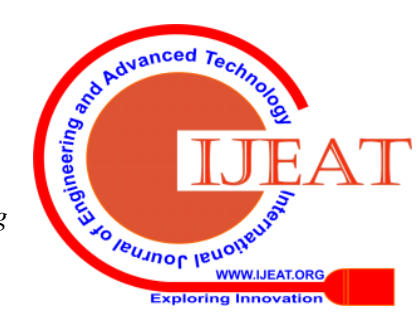




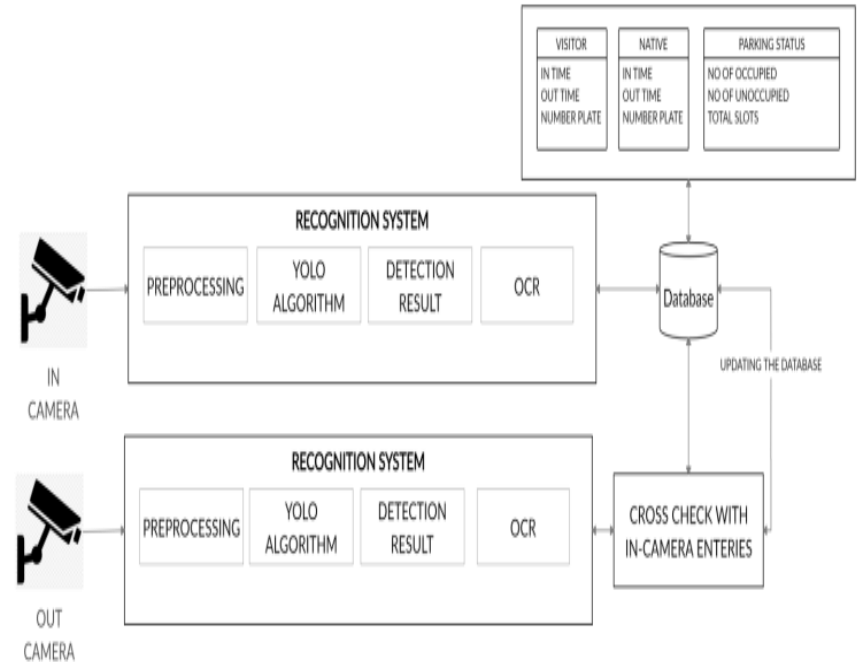

Fig.2. Architecture of Proposed System

\section{LIST OF MODULES}

The proposed system consist of

1. Recognition System
a. Initialization
b. YOLO Algorithm
c. Detection Result
d. OCR

2. Cross Checking

3. Database

\section{RECOGNITION SYSTEM}

\section{A. Intialization}

The raw input image is not fed into the network. Hence the image was preprocessed before, it is known as Initialization.

The preprocessing involves

- Read image

- Resize image

- Remove noise

Once the preprocessing is done, the input is fed into the network.

\section{B. Yolo Algorithm}

The detection of the number plate from the input is the first step in the process of the system. For this purpose we are using the YOLOv3 (You Look Only Once) for the detection of the location of the license plate from the image.

It processes the image at $30 \mathrm{FPS}$ and has a mAP of $57.9 \%$. The preprocessed image victualed into the network (YOLO). The network fed with the input image, it extract the feature maps of the input and process it for the detect the location of the proposed object. After the process it produces the confidence score of the object and indicate the object with bounding over it with the detection score.

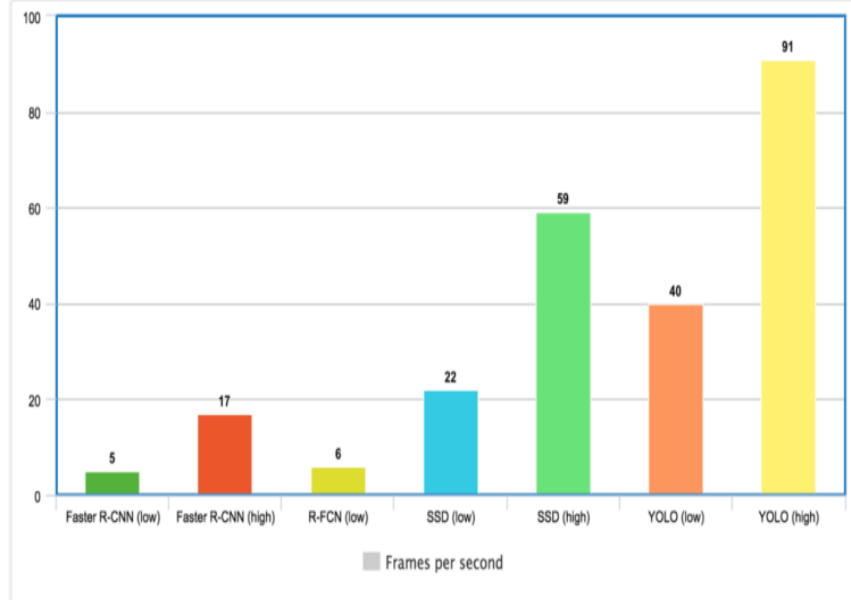

Fig.3. YOLO vs other detectors

From Fig 3 it is clear that YOLO has the higher detection when compared to other detectors such as Faster-RCNN, Fast R-CNN, SSD etc.

\section{Detection Results}

Thus the output from the network produces the final detection result (i.e.) extract the number plate from the image which is integrated with bounding box and classification score.

The mAp and speed comparison of YOLO is plotted in Fig. 3 and it is clear that the proposed algorithm achieved the higher mAp score in the less time. Hence the rate of detection is high when compared to others. However FPN FRCN has the high mAP score it consumes more time compared to YOLOv3.

YOLOv3 is found to be the fastest detector and more accurate. However it has the less detection accuracy than the Fast R-CNN. It performs very well.

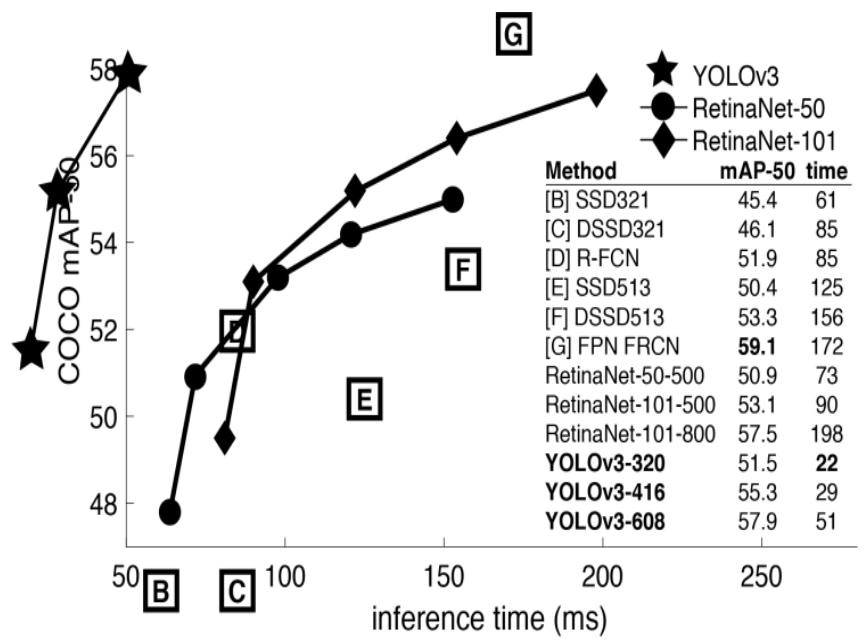

Fig.4. mAP and Speed comparison

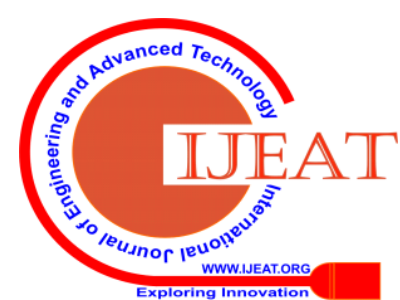




\section{Residential Area Surveillance and Parking System}

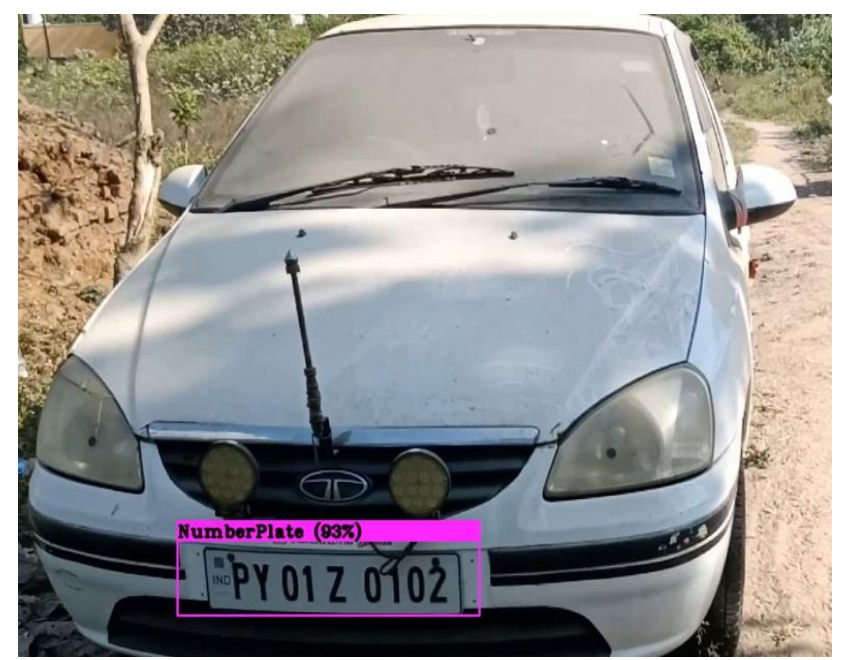

Fig.5. Detection result

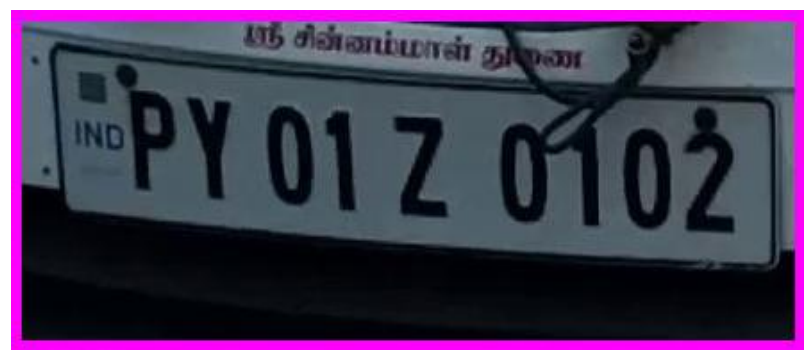

Fig .6.Extracted the detected region

\section{OCR (optical character recognition)}

Python-tesseract is an optical character recognition (OCR) tool used for python.(i.e.), it will recognize and "read" the text detected in the images. The detected number plate is passed to pytessaract engine which converts the optical character in the license plate to the plain text.

\section{(1) text = pytesseract.image_to__tring(Image.open('/content/Untitled.png' $)$ ) print(text) \\ LF PY 0120102}

Fig.7. OCR of license plate

\section{CROSS CHECKING}

It compares the number plate in-camera ingressions and out-camera ingresses in the database for the out time of the concrete conveyance and to update the parking status.

\section{DATABASE}

The database maintains the individual table for the Visitor, Natives, and the Parking status.

The visitor table consists of

- IN-TIME

- OUT-TIME

- NUMBER PLATE ENTRY

The Native table consists of

- NAME OF THE RESIDENT

- IN-TIME

- OUT-TIME

- NUMBER PLATE ENTRY
The Parking status table consists of

- NUMBER OF OCCUPIED SLOT

- NUMBER OF UNOCCUPIED SLOT

TOTAL SLOT

\section{CONCLUSION}

The proposed model for ANPR based residential area surveillance and parking system can able to detect and recognize the number plate of the vehicle which used for automatic authorized or unauthorized vehicle identification system in the residential societies. Implementing this method will reduce the manpower involved in the surveillance. It helps to monitor the parking area.

It assures the surveillance for 24/7. Time taken for detection is reduced drastically which is yet another advantage of this system.

In future it can be enhanced to tracking the vehicle and extracting the more information about the unauthorized visitors. Further it can be used in the areas such as toll gates, Vehicle theft Identification etc.

\section{REFERENCES}

1. Weidong Min, XiangpengLi, Qi Wang ,QingpengZeng ; Scale-Adaptive License Plate Detection

2. T. Hassner, S. Harel, E. Paz, R. Enbar, Object Detection Based on YOLO Network

3. Y. Liu, I. J. Wassell Investigation of Efficient New approach to vehicle license plate location based on new model YOLO-L and plate pre-identification System E. Elhamifar, R. Vidal, V Faster R-CNN: Towards Real-Time Object Detection with Region Proposal Networks -based object detection and tracking for autonomous navigation of underwater robots images.

4. W. Deng, J. Hu, J. Guo, Extended SRC: Scale-aware Fast R-CNN for Pedestrian Detection, E. Elhamifar, R. Vidal, Template-based learning of grasp selection.

5. J. Xing, J. Li, Z. Xie et al., "Research and implementation of an improved radon transform for license plate recognition", IEEE Int. Conf. on Intelligent Human Machine Systems and Cybernetics, pp. 45-48, 2016.

6. K. Deb, K.H. Jo, "HSI color based vehicle license plate detection", Int. Conf. on Control Automation and Systems.

7. D. Zheng, Y. Zhao, J. Wang, "An efficient method of license plate location"

8. P.I. Reji, V.S. Dharun, "License plate detection and recognition using vertical based edge detection algorithm and radial basis function neural network".

9. T. Ojala, M. Pietikainen, T. Maenpaa, "Multiresolution gray-scale and rotation invariant texture classification with local binary patterns."

10. R. Wang, N. Sang, R. Huang et al., "License plate detection using gradient information and cascade detectors"

\section{AUTHORS PROFILE}

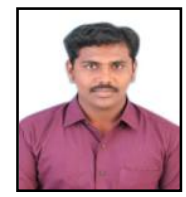

P. Mathivanan is currently working as Assistant Professor in Department of Information Technology, Manakula Vinayagar Institute of Technology, Madagadipet, Pondicherry. He has 9 years of teaching experience and taught more than 12 subjects for B.Tech., Students of Information Technology and Engineering discipline. He Completed M.Tech Information Technology at Sathyabama University. He is a Life time member of Indian Society of Technical Education (ISTE) and International Association of Engineers (IAENG)

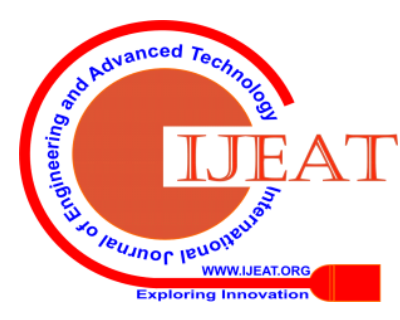




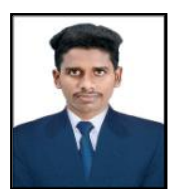

G. G. Amarnath is an aspiring engineer pursuing his bachelor's under the stream of Information Technology in Manakula Vinayagar Institute of Technology, with an unquenchable desire to serve the society through his innovations who in this pursuit has developed a system based on the problem statement provided in the "SMART INDIA HACKATHON 2020" for the residential area surveillance and parking.

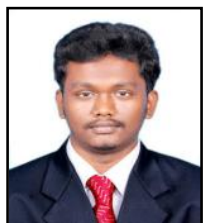

R. Anantharaman is an aspiring engineer pursuing his bachelor's under the stream of Information Technology in Manakula Vinayagar Institute of Technology, with an unquenchable desire to serve the society through his innovations who in this pursuit has developed a system based on the problem statement provided in the "SMART INDIA HACKATHON 2020" for the residential area surveillance and parking.

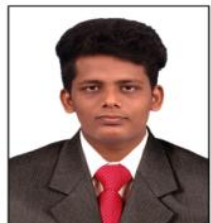

M. Bharath Kumar is an aspiring engineer pursuing his bachelor's under the stream of Information Technology in Manakula Vinayagar Institute of Technology, with an unquenchable desire to serve the society through his innovations who in this pursuit has developed a system based on the problem statement provided in the "SMART INDIA

HACKATHON 2020" for the residential area surveillance and parking.

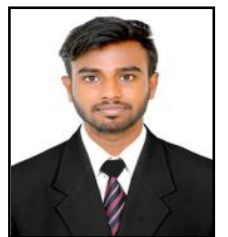

A. Joseph Charles is an aspiring engineer pursuing his bachelor's under the stream of Information Technology in Manakula Vinayagar Institute of Technology, with an unquenchable desire to serve the society through his innovations who in this pursuit has developed a system based on the problem statement provided in the "SMART INDIA HACKATHON 2020" for the residential area surveillance and parking.

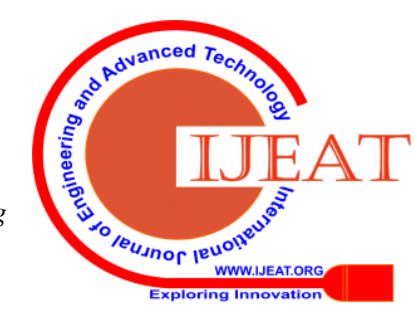

